\title{
Effect of Thermal and Mechanical Loadings on the Residual Stress Field in a Nickel Based Superalloy using X-Ray Laue Microdiffraction
}

\author{
Gader Altinkurt 1,2,a ${ }^{*}$, Mathieu Fèvre ${ }^{1, b}$, Guillaume Geandier ${ }^{2, \mathrm{c}}$, \\ Odile Robach ${ }^{3,4, d}$, Soufiane Guernaoui ${ }^{2, e}$ and Moukrane Dehmas ${ }^{2, f}$ \\ ${ }^{1}$ LEM, UMR 104 CNRS-Onera, Châtillon, France \\ ${ }^{2}$ IJL, UMR 7189 CNRS-Université de Lorraine, Nancy, France \\ ${ }^{3}$ CRG-IF BM32 at ESRF Grenoble, France \\ ${ }^{4}$ CEA, INAC-SP2M, Grenoble, France \\ a gader.altinkurt@onera.fr, ${ }^{\mathrm{b}}$ mathieu.fevre@onera.fr, ${ }^{\mathrm{c}}$ guillaume.geandier@univ-lorraine.fr, \\ d odile.robach@cea.fr, ${ }^{e}$ soufiane.xr@gmail.com, ${ }^{f}$ moukrane.dehmas@univ-lorraine.fr
}

Keywords: Laue Microdiffraction, Residual Deviatoric Strain, Shot-Peening, Fatigue, Coarse-Grained Crystal, Nickel Based Superalloy

\begin{abstract}
The shot-peening operation is used to improve the fatigue lifetime of mechanical components through the introduction of compressive residual stresses and plastic deformation in a surface layer. In this study, the Laue microdiffraction technique is used to investigate deviatoric strain fields caused by the shot-peening operation and their redistribution after fatigue testing in a nickel-based polycrystalline superalloy with a $40 \mu \mathrm{m}$ average grain size.
\end{abstract}

\section{Introduction}

Nickel-based polycrystalline superalloys elaborated by powder metallurgy are used in high pressure turbine discs of aircraft engines. Specific compositions, microstructures and mechanical treatments are developed to enhance crack growth, creep and fatigue resistance during service. The shot-peening process is used extensively to delay crack initiation in the surface layer of components by introducing compressive residual stresses and plastic deformation. The determination of such stresses is generally performed with diffraction based techniques using the $\sin ^{2} \Psi$ method and the effect of temperature and fatigue conditions on stresses relaxation has been deeply investigated in fine-grained microstructures. Most outcomes are associated with macroscopic properties of the average alloy. At the grain scale, electron backscattered diffraction and transmission electron microscopy were employed to characterize plastic deformation and dislocation densities after the shot-peening operation [1]. Even if compressive residual stresses have been relaxed, high dislocation densities persist in the microstructure providing hardening and resistance. A complete understanding of the relationship between the microstructure (precipitate and grain sizes) and residual stresses is still missing. With the Laue microdiffraction technique, stress states and geometrically necessary dislocation densities can be investigated at the grain and phase scales. The technique has already been used to understand the dislocation mechanism in grains under a tensile loading [2], near to grain boundary during a fatigue loading [3] or in zone heavily deformed by friction stir welding [4]. Local deviatoric strain or stress tensors have been also determined in areas close to scratches [5] and cracks [6] or under a tensile loading [7]. The Laue microdiffraction technique has not been employed to characterize strain fields in shot-peened nickel-based superalloys. This method is in principle a suitable tool for macroscopic and microscopic stress determination in coarse grained microstructures since the $\sin ^{2} \Psi$ method is not well adapted to investigations in such microstructures. 
In this paper, deviatoric strain fields generated by the shot-peening operation and then redistributed by a low-cycles fatigue test at $450{ }^{\circ} \mathrm{C}$ are characterized in a nickel-based superalloy with a $40 \mu \mathrm{m}$ average grain size.

\section{Experimental conditions}

Sample features. Cylindrical specimens were removed using Electro Discharge Machining in a sector of a turbine disk of the N18 superalloy [8] produced at Snecma (SAFRAN group). The microstructure consists in $\gamma^{\prime}$ hardening precipitates with a simple cubic structure embedded in a $\gamma$ matrix phase with a face-centered cubic structure. A coarse grained microstructure with one population of $\gamma^{\prime}$ precipitates is obtained after a supersolvus solution treatment ( 4 hours at $1205^{\circ} \mathrm{C}$ and air quench) followed by an ageing treatment $\left(1 \mathrm{~h}\right.$ at $900^{\circ} \mathrm{C}$ and air quench). At the millimetre scale, the analysis of the Electron Back Scattered Diffraction (EBSD) images acquired in the Scanning Electron Microscope (SEM) shows an average $\gamma$ grain size of $40 \mu \mathrm{m}$ with a wide distribution ranging from $5 \mu \mathrm{m}$ to $200 \mu \mathrm{m}$ (Fig. 1b). At the micrometer scale, SEM micrographs show cubic shape $\gamma^{\prime}$ precipitates with an average size close to $200 \mathrm{~nm}$ homogeneously distributed in the $\gamma$ matrix (Fig. 1c). In order to simplify the x-ray data analysis and to dissociate physical mechanisms in such materials, this microstructure sensibility differs from those employed in aircraft engines $(5-20 \mu \mathrm{m}$ grain sizes and three populations of $\gamma^{\prime}$ precipitates [8]). Three $6.22 \mathrm{~mm}$ in diameter fatigue test specimen were then machined. One was used as a reference. One was subjected to shot-peening, one to shot-peening and interrupted fatigue testing. The peening operation was done using an ultrasonic technique with $3 \mathrm{~mm}$-diameter $100 \mathrm{Cr} 6$ steel shots during 1080s. The fatigue test was performed at imposed deformation $\left(\Delta \varepsilon / 2=0.5 \%, \mathrm{R}_{\varepsilon}=0\right)$ at $450^{\circ} \mathrm{C}$ during 300 cycles. $10 \mathrm{~mm}$ height samples were finally cut perpendicular to the shot-peened surface in the gauge length. X-ray measurements were performed in a circular cross section which has been mechanically and chemically polished to reduce stresses introduced by the cutting process.
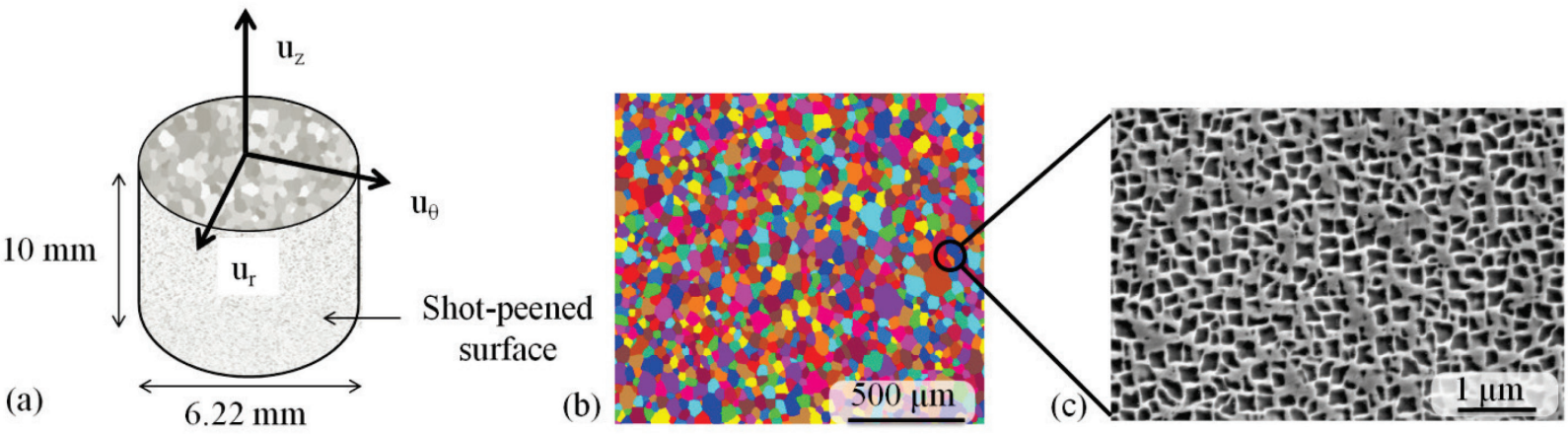

Fig. 1: (a) sample geometry with the local cylindrical coordinate system $\left(\overrightarrow{u_{r}}, \overrightarrow{u_{\theta}}, \overrightarrow{u_{z}}\right)$. (b) EBSD map revealing grains $\gamma$ (random colours). (c) SEM micrograph showing $\gamma$ ' precipitates (dark grey) embedded in the $\gamma$ matrix (light grey).

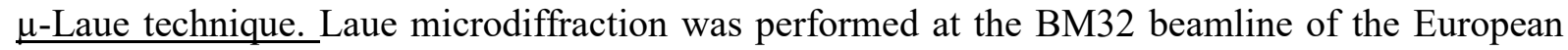
Synchrotron Radiation Facility at Grenoble (France). A polychromatic beam with a $5-17 \mathrm{keV}$ energy spectrum is focused to a cross section of $0.35 \times 0.6 \mu \mathrm{m}^{2}$ at the sample position. Emerging X-rays are collected on a CCD 2D detector oriented in $90^{\circ}$ with respected to the incident beam in reflection geometry (see Ref. [9] for a more detailed description of the setup). A xyz motorized sample stage enables to record Laue patterns arising from different locations at the sample surface. With this setup, the characteristic penetration depth of the beam in the material ranges from $4 \mu \mathrm{m}$ to $15 \mu \mathrm{m}$ and up to one frame per 5 second can be acquired. Due to the grain size and the cross section of the beam, our samples behave like single crystals and the Laue pattern consists in spots. The Miller indices of each spot can be retrieved from the crystal symmetry and peak positions in pattern. Based on the formulation proposed by Hung and Ice [10], the indexation of four diffraction peaks permits to determine the orientation of the crystal lattice regarding to the coordinate system of the experiment 
and five of the six unit cell parameters: two lengths ratio and three angles usually denoted by $\alpha, \beta, \gamma$ and $\mathrm{b} / \mathrm{a}, \mathrm{c} / \mathrm{a}$ respectively. The knowledge of these parameters for a non-deformed structure $\left(a_{0}, b_{0}, c_{0}, \alpha_{0}, \beta_{0}, \gamma_{0}\right)$ enables to determine the deviatoric elastic strain tensor $\varepsilon^{d}$ within the small transformation approximation. Its coefficients are given by:

$$
\varepsilon_{\mathrm{ij}}^{\mathrm{d}}=\varepsilon_{\mathrm{ij}}-\operatorname{Tr}(\varepsilon) \cdot \delta_{\mathrm{ij}} / 3 \quad \mathrm{i}, \mathrm{j}=1,2,3
$$

where $\varepsilon$ is the total strain tensor and $\delta_{\mathrm{ij}}$ the Kronecker delta function. $\varepsilon^{\mathrm{d}}$ is associated with the distortion of the crystal unit cell. When the stiffness tensor of the single crystal is known, the deviatoric stress tensor can be deduced by applying the generalized Hooke's law.

\section{Data processing}

Due the highly coherent interfaces (lattice mismatch $<10^{-3}$ ), fundamental reflections $(h, k, l$ with the same parity) correspond to the convolution of the intensities belong to the $\gamma$ and $\gamma^{\prime}$ phase whereas superstructure reflections ( $h, k, l$ with different parities) correspond only to the contribution of the $\gamma^{\prime}$ phase intensities. Laue patterns provide thus quantitative information about the average alloy $\left(\gamma+\gamma^{\prime}\right)$ and the $\gamma$ ' phase. Data analysis has been performed using the XMAS [11] and LaueTool [12] softwares. The main output of the calculations is the coordinates of the reciprocal lattice vectors in a given system of coordinates (sample, laboratory) for each crystallite founded in the Laue pattern. This is then used to determine the crystal orientation, its unit cell parameters and finally deviatoric strain and stress components. The strain-free lattice-parameters of the $\gamma$ and $\gamma^{\prime}$ phases have been determined for the fine grain N18 alloy [8]. A Vegard's law is used to determine the strain free lattice-parameter associated with the average alloy $\left(\mathrm{a}_{0}=0.35917 \mathrm{~nm}\right)$. With the Laue technique, the strain sensitivity can be $5.10^{-5}$ when optimal conditions are reached [13] but generally, it is closer to $1-2.10^{-4}[5,10]$. In this study, the assessment of uncertainties in a sample which has not been subjected to shot-peening results in a $2.10^{-4}$ uncertainty on strain values.

\section{Results and discussions}

In the following, results associated with the analysis of the fundamental reflections $\left(\gamma+\gamma^{\prime}\right)$ are presented. The crystal orientation is expressed with respect to the fixed laboratory coordinate system within the axis-angle representation. Based on the sample geometry and the applied external loadings, the deviatoric strain components $\varepsilon^{\mathrm{d}}{ }_{\mathrm{ij}}$ are given with respect to the local cylindrical coordinate system $\left(\overrightarrow{u_{r}}, \overrightarrow{u_{\theta}}, \overrightarrow{u_{z}}\right) \cdot \overrightarrow{u_{r}}, \overrightarrow{u_{\theta}}$ correspond to radial and circumferential axis and $\overrightarrow{u_{z}}$ is the axis aligned with the free surface normal (Fig.1a). The single-crystal elastic constants of the N18 are unknown. To avoid bad physical interpretations, only strain tensor components are discussed below. Since the deviatoric strain components associated with the $\overrightarrow{u_{z}}$ direction could be affected by the presence of the free surface, only $\varepsilon_{\mathrm{rr}}^{\mathrm{d}}, \varepsilon^{\mathrm{d}}{ }_{\theta \theta}$ and $\varepsilon_{\mathrm{r} \theta}^{\mathrm{d}}$ are discussed below. Orientation and strain xy maps are obtained from meshes with steps corresponding to the beam size.

Reference sample. Measurements in the sample which has not been subjected to shot-peening have been realized on a $1 \times 3 \mathrm{~mm}^{2}$ area. No strain heterogeneities between identified grains were observed. Strain components smoothly evolve with values close to the sensitivity limit of the technique $\left( \pm 2.10^{-4}\right)$ excepting for the $\varepsilon^{\mathrm{d}}{ }_{\mathrm{zz}}$ component which is between $-3.10^{-4}$ and $-6.10^{-4}$. This is probably due to the surface polishing realized during the sample preparation. Such values of deviatoric strains have also been obtained by Chao et al [6] with the same technique in a nickel-based Alloy 600 sample before tensile deformation. The analysis also shows that strains in the order of $\pm 1.5 \times 10^{-3}$ caused by the grinding machine used develop over a layer with a thickness lower than 100 $\mu \mathrm{m}$.

Effect of shot-peening. Measurements in the sample which has been subjected to shot-peening have been realized on a $1.02 \times 0.225 \mathrm{~mm}^{2}$ area close to its edge. The xy-orientation map and the maps associated with the components of the deviatoric strain tensor are represented in figure 2 (a-d). Lines in figure $2 \mathrm{a}$ correspond to radius contours and the edge of the sample is located at the right side of the figure (last contour line). In the first $300 \mu \mathrm{m}$ wide area close to sample edge (white color), the 
material hardening introduced by the shot-peening has caused large intensity spread in the Laue pattern. It was not possible to accurately quantify strain tensor components. Figures $2 \mathrm{~b}$ to $2 \mathrm{~d}$ show different components of the strain field. Along the average peening direction $\overrightarrow{u_{r}}$ (Fig. 2b), the deviatoric strain component strongly depends on the distance from the sample centre (negative for $2.0 \mathrm{~mm}<\mathrm{x}<2.5 \mathrm{~mm}$ and positive for $2.5 \mathrm{~mm}<\mathrm{x}<2.7 \mathrm{~mm}$ ) and has a less pronounced variations along contours. Strain amplitudes range is from $-4.10^{-3}$ to $4.5 \times 10^{-3}$. Along the circumferential direction $\overrightarrow{u_{\theta}}$ (Fig. 2c), the deviatoric strain component has also this dependency with a negative sign. Some variations can be observed close to several grain boundaries. Strain amplitudes range is from $4.5 \times 10^{-3}$ to $1.10^{-3}$. In figure $2 \mathrm{~d}$, the $\varepsilon_{\mathrm{r} \theta}^{\mathrm{d}}$ component has a heterogeneous behaviour with strain levels much more dependent on the microstructure and a range from $-2.10^{-3}$ to $2.10^{-3}$. For all components, we clearly observe that after shot peening, high shear strains take place deeply in the material. Indeed, at a distance of $1 \mathrm{~mm}$, all components differ significantly from zero. In order to compare Laue microdiffraction results to those which could be obtained in the laboratory with a beam section close to square millimetres, strain values can be averaged along the radius contour lines of figure $2 \mathrm{a}$. Results are represented as a function of the distance from the shot-peened edge in figure $3 \mathrm{a}$. Because of the dependency of the deviatoric strain at this scale, this representation is justified. The $\varepsilon_{\text {rr }}^{\mathrm{d}}$ and $\varepsilon^{\mathrm{d}}{ }_{\theta \theta}$ components follow trends described previously. A large variation of strains is visible in a layer located between $0.3 \mathrm{~mm}$ and $0.6 \mathrm{~mm}$ depths and they evolve smoothly further. The $\varepsilon_{\mathrm{r} \theta}^{\mathrm{d}}$ component is close to zero for all depths and the presence of strong local heterogeneities is not discernable with this average.

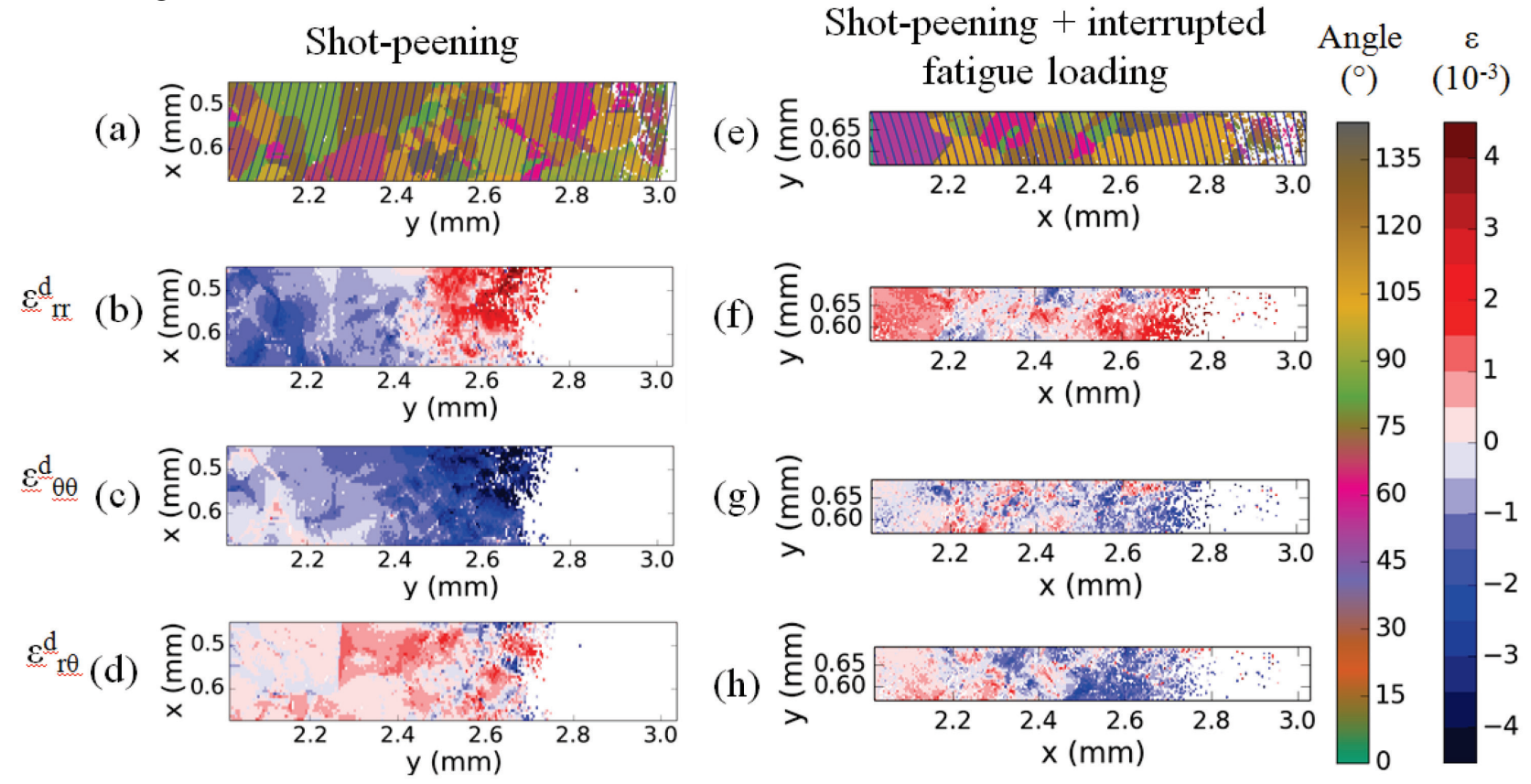

Figure 2: Local orientation and deviatoric elastic strain component maps for a sample which has been subjected to shot-peening on the left and to shot-peening and an interrupted fatigue test at $450^{\circ} \mathrm{C}$ on the right. Orientation maps are determined within the axis-angle representation.

Effect of fatigue. Figure 2e-h represents the orientation and the deviatoric strain maps obtained from measurements in the sample which has been shot-peened and then subjected to low-cycle fatigue at $450^{\circ} \mathrm{C}$ during 300 cycles. The investigated area is $1.01 \times 0.125 \mathrm{~mm}^{2}$ close to a sample edge. The deviatoric strain fields observed just after the shot-peened in figures $2 \mathrm{~b}$ to $2 \mathrm{~d}$ have been strongly modified by the thermal and mechanical loading. All components exhibit positive and negative heterogeneous strains in the whole area with amplitudes comparable to what has been determined previously $\left(-3.10^{-3}<\varepsilon^{d}<4.10^{-3}\right)$. By contrast with the shot-peened state, the axial and the circumferential deviatoric strains are independent on the distance from the sample edge and more 
influenced by each grain features. Strain values averaged along the radius contour lines are represented as a function of the distance from the shot-peened edge in figure $3 \mathrm{~b}$. On the few considered grains, the $\varepsilon_{r \theta}^{\mathrm{d} \theta}$ component behaves similarly for these two states. However, some relaxation is visible for the two other components after fatigue. To have a better statistic and probe a large number of grains, strain profiles from a $2 \times 2 \mathrm{~mm}^{2}$ map (not shown) with a smaller spatial resolution are plotted in figure $3 \mathrm{c}$. At this scale, all components differ significantly from zero up to a distance of $0.4 \mathrm{~mm}$ from the sample edge. The highest strain amplitudes are close to $1 \times 10^{-3}$ and take place close to the shot-peened surface. Most of the fluctuations visible in figure $3 \mathrm{~b}$ are removed by the averaging procedure and the $\varepsilon_{r \theta}^{\mathrm{d} \theta}$ component behaves differently close to the sample edge in figure $3 \mathrm{~b}$ and $3 \mathrm{c}$. These results highlight the fact that large local strains occur in the microstructure even though no strain is determined with a method involving averages on many grains as with the $\sin ^{2} \Psi$ method. Consequently, a good understanding of the role of the microstructure on the fatigue properties of materials requires investigations at the grain scale.

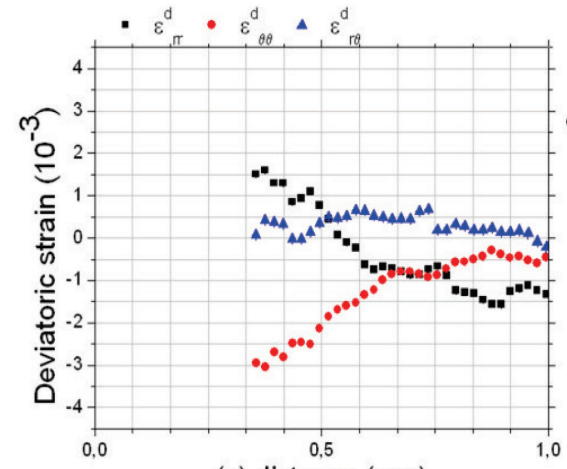

(a) distance $(\mathrm{mm})$

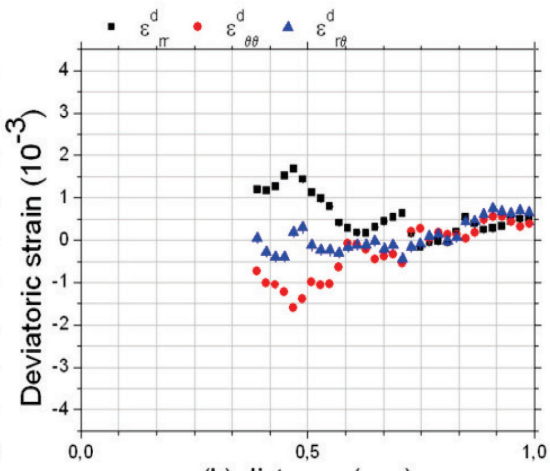

(b) distance $(\mathrm{mm})$

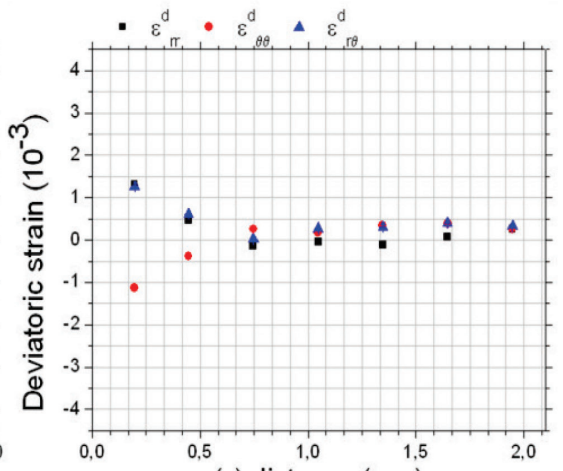

(c) distance $(\mathrm{mm})$

Figure 3: Deviatoric strain components obtained after averaging along radius contour lines (a) after the shot-peening operation, (b) and (c) after the interrupted fatigue test at $450^{\circ} \mathrm{C}$. Distances are given from the shot-peened sample edge.

\section{Conclusions}

The Laue microdiffraction technique has been used to investigate deviatoric strain fields in nickelbased superalloy samples with a coarse grained microstructure and subjected to ultrasonic shotpeening and fatigue testing at $450^{\circ} \mathrm{C}$. This method is suitable for investigations in coarse grained microstructures as an alternative to conventional techniques and provides additional information at the grain scale. The method should able to study the plastic deformation induced by shot-peening with the study of peaks shape. It should able to provide new data on the influence of the $\gamma^{\prime}$ precipitates on internal stresses in superalloys. However, information from the highly strained 300 $\mu \mathrm{m}$ thick region close to the surface could not be obtained as Laue patterns could not be indexed with a good accuracy. Moreover, the mapping of the full strain tensor is still a challenging task.

Measurements at the grain scale have shown that residual elastic strains with amplitudes ranging from -4 to $4.10^{-3}$ take place at depths larger than $1 \mathrm{~mm}$. The redistribution of strain fields associated with the shot-peening operation was also clearly observed after 300 cycles of fatigue at $450^{\circ} \mathrm{C}$. In particular, the dependence of axial and circumferential strains with the distance from the shot-peened surface observed just after the shot-peening operation has completely disappeared after fatigue leaving to heterogeneous positive and negative fields with a strong dependence on the underlying microstructure.

\section{Acknowledgments}

This work benefited from the support of the project REMEDDIES ANR-13-BS09-016 of the French National Research Agency (ANR). The experiments were performed on beamline BM32 at the European Synchrotron Radiation Facility (ESRF), Grenoble, France. We are grateful to Local Contact at the ESRF for providing assistance in using beamline BM32. 


\section{Reference}

[1] O.M.D.M. Messe, S. Stekovic, M.C. Hardy, C.M.F. Rae, Characterization of plastic deformation induced by shot-Peening in a Ni-Base superalloy, JOM 66 (2014) 2502-251. https://doi.org/10.1007/s11837-014-1184-8

[2] R. I. Barabash, E.W Huang, J.J. Wall, J.H. Wilkerson, Y. Ren, W. Liu, S.C. Vogel, G.E. Ice, L.M. Pike, P.K. Liaw, Texture crossover: Trace from multiple grains to a subgrain, Mater. Sci. Eng. A528 (2010) 3-10. https://doi.org/10.1016/j.msea.2010.07.035

[3] E.-W. Huang, R.I. Barabash, G.E. Ice, W.J. Liu, Y.-L. Liu, J.-J. Kai, P.K. Liaw, Cyclic-loadinginduced accumulation of geometrically necessary dislocations near grain boundaries in an Ni-based superalloy, JOM 61-12 (2009) 53-58. https://doi.org/10.1007/s11837-009-0181-9

[4] O.M. Barabash, R.I. Barabash, G.E. Ice, Z.Feng, D. Gandy, X-ray microdiffraction and EBSD study of FSP induced structural/phase transitions in a Ni-based superalloy, Mater. Sci. Eng. A524 (2009) 10-19. https://doi.org/10.1016/j.msea.2009.03.086

[5] M.L. Suominen Fuller, R.J. Klassen, N.S. McIntyre, A.R. Gerson, S. Ramamurthy, P.J. King, W. Liu, Texture, residual strain, and plastic deformation around scratches in alloy 600 using synchrotron X-ray Laue micro-diffraction, J. Nucl. Mater. 374 (2008) 482-487. https://doi.org/10.1016/j.jnucmat.2007.10.015

[6] J. Chao, A. Mark, M.L. Suominen Fuller, N.S. McIntyre, R.A. Holt, R.J. Klassen, W.J. Liu, Study of residual elastic- and plastic-deformation in uniaxial tensile strained nickel-based Alloy 600 samples by polychromatic X-ray microdiffraction (PXM) and neutron diffraction methods, Mater. Sci. Eng. A524 (2009) 20-27. https://doi.org/10.1016/j.msea.2009.05.033

[7] J. Chao, M.L. Suominen Fuller, N. Sherry, J. Qin, N. Stewart McIntyre, J. Ulaganathan, A.G. Carcea, R.C. Newman, M. Kunz, N. Tamura, Plastic and elastic strains in short and long cracks in Alloy 600 studied by polychromatic X-ray microdiffraction and electron backscatter diffraction, Acta Mater. 60 (2012) 5508-5515. https://doi.org/10.1016/j.actamat.2012.06.060

[8] S.T. Wlodek, M. Kelly, D. Alden, The Structure of N18, Proceedings of Superalloys 1992 (1992) 467-476. https://doi.org/10.7449/1992/superalloys_1992_467_476

[9] O. Ulrich, X. Biquard, P. Bleuet, O. Geaymond, P. Gergaud, J.S. Micha, O. Robach, F. Rieutord, A new white beam x-ray microdiffraction setup on the BM32 beamline at the Europea Synchrotron Radiation Facility, Rev. Sci. Instr. 82 (2011) 033909-1-6. https://doi.org/10.1063/1.3555068

[10] J. Chung, G. Ice, Automated indexing for texture and strain measurement with broad-bandpass X-ray microbeams, J. Appl. Phys. 86 (1999) 5249-5255. https://doi.org/10.1063/1.371507

[11] N. Tamura, A. A. MacDowell, R. Spolenak, B. C. Valek, J. C. Bravman, W. L. Brown, R. S. Celestre, H. A. Padmore, B. W. Batterman, J. R.J. Patel, Scanning X-ray microdiffraction with submicrometer white beam for strain/stress and orientation mapping in thin films, Synchrotron. Radiat. 10 (2003) 137-143. https://doi.org/10.1107/S0909049502021362

[12] Information on https://sourceforge.net/projects/lauetools/

[13] F. Hofmann, S. Eve, J. Belnoue, J.S. Micha, A. M. Korsunsky, Analysis of strain error sources in micro-beam Laue diffraction, Nuclear Instrum. Methods Phys. Res. A660 (2011) 130-137. https://doi.org/10.1016/j.nima.2011.09.009 